\title{
Tuning Optical Discs for Plasmonic Applications
}

\author{
Burkan Kaplan · Hasan Guner • Ozlem Senlik • \\ Kemal Gurel · Mehmet Bayindir · Aykutlu Dana
}

Received: 9 February 2009 / Accepted: 22 June 2009 / Published online: 4 July 2009

(C) Springer Science + Business Media, LLC 2009

\begin{abstract}
We present simple physical and chemical procedures that allow tuning and modification of the topography of gratings present in optical storage discs into geometries optimal for grating coupled plasmon resonance excitation. After proper metal coating, the tuned surfaces exhibit sharp plasmon resonances that can be excited at wavelengths ranging from $260 \mathrm{~nm}$ to over $2.7 \mu \mathrm{m}$ with relatively high quality factors. As an immediate exemplary application, use of such optimized gratings in aqueous medium for refractive index measurement is demonstrated.
\end{abstract}

Keywords Diffraction gratings •

Surface plasmons $\cdot$ Plasmonics

\section{Introduction}

Plasmonics, a field with important potential technological applications in sensing [1], imaging [2], and information processing [3], has been the subject of intense study in recent decades. Plasmons are resonant interactions of light with materials of finite carrier density

B. Kaplan · H. Guner · O. Senlik · K. Gurel ·

M. Bayindir · A. Dana $(\varangle)$

UNAM-Institute of Materials Science and Nanotechnology,

Bilkent University, 06800 Ankara, Turkey

e-mail: aykutlu@unam.bilkent.edu.tr

M. Bayindir

Department of Physics, Bilkent University,

06800 Ankara, Turkey

e-mail: bayindir@nano.org.tr and mobility. Such resonant effects can be observed in metal films on dielectrics [4], in chemically synthesized metal nanostructures [5], and in lithographically defined metal nanostructures [6,7] and arrays [8], to name a few. The resonant fields are known to be enhanced in comparison to the vacuum field, and resonance conditions are highly sensitive to surrounding dielectric properties [9]. Also, the fields can be confined to much smaller dimensions than free space wavelengths, with propagation lengths of up to hundreds of micrometers for optimal configurations [10]. The mentioned properties make plasmonic devices appropriate for a variety of applications in sensing, optics, and optoelectronics [11]. Due to these potentials, plasmonics continues to draw attention and increasingly wider availability of high precision fabrication and characterization methods for submicron metallic structures allows further advances in the field of plasmonics. For the fabrication and utilization of plasmonic structures, there are a number of methods where certain geometric properties are dominant in determination of the conditions for resonant coupling. Bulk plasmonic excitations, surface plasmon polaritons, localized surface plasmon resonances, slitgrooves, and grating-coupled plasmon resonances are a few of the names used to distinguish dominant features of structures displaying plasmonic effects [12-14].

In this paper, we present a simple method to convert optical discs (CD, DVD, and Blu-Ray) into geometries optimal for the study of high-quality factor grating coupled plasmon resonances (Fig. 1a, b). Previously, CDs and DVDs have been used in experiments involving grating coupled plasmons $[15,16]$. However, to observe sharp resonances, the depth and shape of corrugations must lie within a restricted range. A sharper resonance improves the overall sensitivity of plasmonic devices 


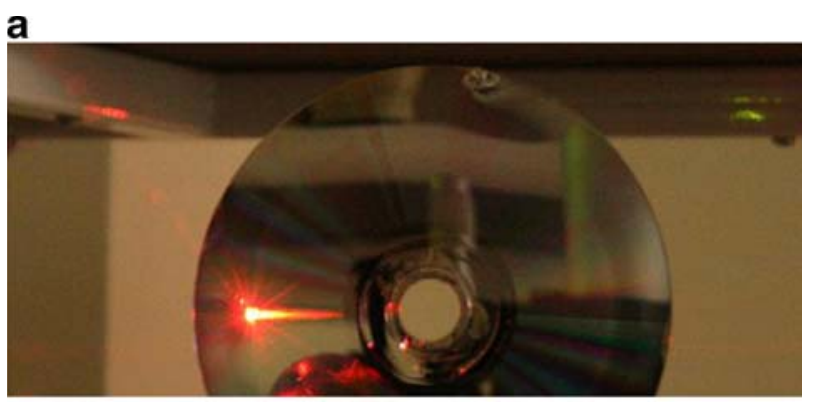

b

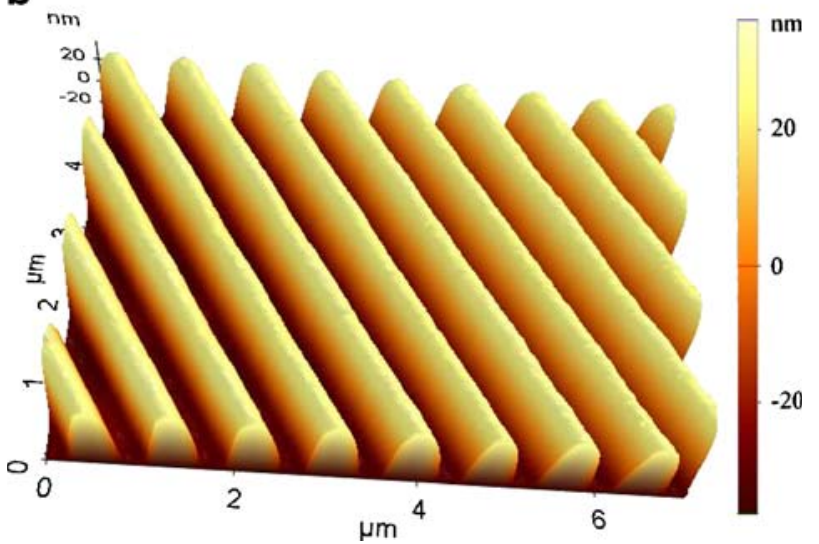

Fig. 1 a Plasmonic excitation observed on a silver-coated DVD surface. The observed coupling occurs at a single polarization and at a specific angle of incidence. b Surface topography by AFM shows the grating structure with a period $\Lambda=740 \mathrm{~nm}$ on the DVD

for biomolecular sensing. Moreover, high-Q resonances result in higher field enhancement factors, which is important in a number of applications, such as surfaceenhanced Raman scattering [17].

On a smooth planar interface of a metal and a dielectric, the surface plasmon polariton (SPP) wave vector is given by

$k_{\mathrm{SPP}}=\frac{\omega}{c} \sqrt{\frac{n_{1}^{2} n_{2}^{2}}{n_{1}^{2}+n_{2}^{2}}}$,

where $n_{1}$ is the complex index of refraction of the dielectric medium and $n_{2}$ is that of the metal layer. Using a prism, free space light can be coupled into the SPP mode. However, at the biomolecular sensing applications, careful choice of materials, excitation wavelength, and geometric configuration have critical importance for optimal excitation. In the presence of a sinusoidal surface corrugation, the coupling condition is modified by the pattern of the grating, and a plane wave approaching the surface at a particular angle of incidence can resonantly couple to the plasmonic excitation. The additional parameter introduced, namely, the grating period, relaxes the matching condition and gives greater freedom for the choice of other parameters. In this case, the angle for resonant coupling is given by [18]

$\Theta_{R}=\arcsin \left[\operatorname{Re}\left(-\sqrt{\frac{n_{1}^{2} n_{2}^{2}}{n_{1}^{2}+n_{2}^{2}}}\right) \pm m \frac{\lambda}{n_{1} \Lambda}\right]$,

where $\Lambda$ is the grating period and $m$ is a positive integer. Resonant coupling to the plasmon mode occurs for TM polarization only, where the grating direction is perpendicular to the plane of incidence.

Most commercially available optical discs have corrugated imprinted surfaces protected by external coatings. By exposing the surfaces, simple chemical procedures can be used to tune the surface profile to optimal geometries for sharp and deep plasmon resonant peaks. The depth and shape of the grating affects the width (apparent quality factor) of the plasmonic resonance. Also, the amplitude and relative phases of spatial Fourier components of the grating are important in the determination of the reflection spectrum of such gratings [6, 7]. For higher peak absorption, which also means larger field enhancement due to stronger plasmon coupling, the factors that need to be tuned are the depth and shape of the gratings. In our case, we used rigorously coupled wave analysis to show the dependence of peak absorption and quality factor on grating depth for sinusoidal grating profile and for a quasisinusoidal profile commonly observed in DVDs. Results of calculations are shown in Fig. 2a. It is seen that the peak absorption is dependent on the sinusoidality of the grating as well as the grating depth. For purely sinusoidal gratings, unity absorption is possible at normal incidence. For a grating with higher-order harmonics (as in a rectangular profile grating), the peak absorption may never reach unity for certain angles of incidence. Normal incidence of a rectangular profile is shown in Fig. 2a, where peak absorption cannot exceed 0.65 due to coupling of different plasmon modes. However, as the angle is a few degrees away from normal incidence, the absorption can still be close to unity for optimal grating depths, due to different dispersion of plasmon resonances associated with higher-order harmonics and the fundamental harmonic. Also, presence of different spatial harmonics of the grating profile causes shifts in the peak wavelength as denoted in the legend of Fig. 2a. The widths of resonances also depend on the grating depth, as shown in Fig. 2b. Gratings with depths of 20 to $60 \mathrm{~nm}$ result in sharp resonances with full-width-at-halfmaxima less than $10 \mathrm{~nm}$. Unetched optical disc profiles (which typically have $100 \mathrm{~nm}$ or more depth for $\mathrm{CD}$ and DVDs) are not optimal for observing sharp and strong resonances. In most applications, it is desirable to have 


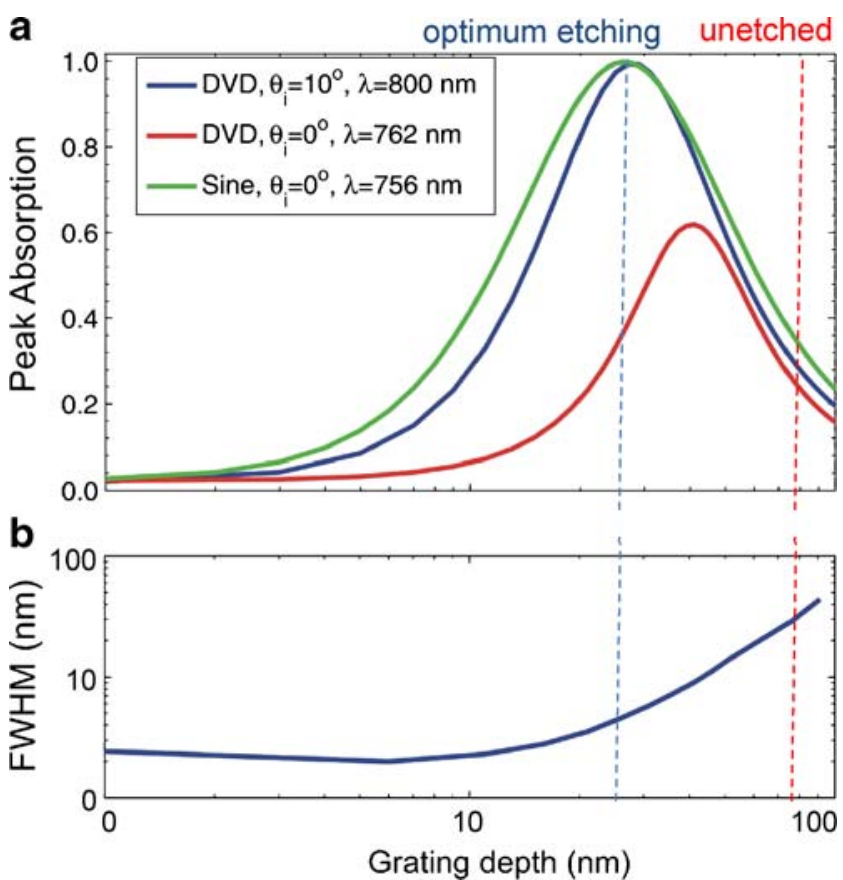

Fig. 2 a Theoretical peak absorption due to grating coupled plasmon resonance on silver-coated gratings on DVD with period $\Lambda=740 \mathrm{~nm}$ at various angles of incidence as a function of grating depth and shape. Peak absorption occurs at different wavelengths depending on the angle of incidence. b Full-widthat-half-maximum of the absorption peak for a grating with a DVD profile. Curves are calculated using rigorously coupled wave analysis

clean, sharp, and strong resonances; the grating depths need to be reduced to an optimal depth; and shapes must be made converged to a sinusoid.

\section{Modification of optical disc profiles}

Optical discs (CD, DVD, and Blu-Ray) come in a wide variety of microscopic structures and use a wide range of materials. In this work, a relatively large number of discs (CD-R, CD-RW, DVD-R, DVD-RW, Blu-Ray recordable) from different manufacturers are studied. The rewritable discs (CD-RW and DVD-RW) generally use a hard chalcogenide material as the recording medium, and Blu-Ray write-once discs also use a multilayer hard coating. However, the write-once CD-R and DVD-R discs use a dye layer as the recording medium that can be dissolved in weaker solvents. Therefore, we focused on write-once discs for surface modification. Since different optical disc technologies have different layer structures, slightly different methods were used to prepare the grating substrates. CDs have a thin organic coating on top of a metal coating covering the dye layer and the grating, whereas the DVD-R structure consists of two polycarbonate discs with spiral grating surfaces in between. Blu-Ray discs have a relatively thin layer of transparent coating covering the grating layer. Towards the edges of the discs, the track spirals are well approximated by gratings with periods of $\Lambda=$ $1600 \mathrm{~nm}$ for the CD-R, $\Lambda=740 \mathrm{~nm}$ for the DVD-R, and $\Lambda=320 \mathrm{~nm}$ for Blu-Ray.

For the case of the CD-R, the top protective layer was peeled off by exfoliation using a tape after delineation of the surface with a sharp cutter. After peeling off the top surface, the dye layer was removed by washing it with isopropanol. This procedure was observed not to change the corrugations on the disc but to completely remove the dye. After cleaning, a 4:1 mixture of isopropanol and acetone was used to etch the grating to the desired depth. Typical etch times were found to be $10 \mathrm{~s}$ to a few minutes. Slight agitation during etching was observed to be important for uniform and repeatable etching. Different brands of commercial CDs were used, and slight differences in corrugation depth and grating shape, as well as differences in etch rates, were observed. The surfaces are then coated with silver films of 50 to $100 \mathrm{~nm}$ thickness using thermal vacuum evaporation. In the case of DVD-R, due to the thick layers that protect the recording layer, instead of exfoliation by tape, the inner surface was exposed by first mechanically cutting a notch into the side of the disc and then by applying mechanical force to peel the two sides apart. The initial cut provides a location for separation, which can be initiated from the side by a sharp cutter. The same procedure that was used for $\mathrm{CD}-\mathrm{R}$ was also used for cleaning, etching, and metal coating of DVD-R discs. Exposing the Blu-Ray disc gratings was done by peeling off the transparent thin coating with tweezers after cutting with scissors. Unlike CD-R and DVD-R, Blu-Ray discs have a nearly sinusoidal grating shape with $20 \mathrm{~nm}$ grating depth, which we do not need to modify, and a $320-\mathrm{nm}$ grating period. This grating period brings plasmon resonances at around $320 \mathrm{~nm}$ wavelength depending on the angle of incidence. Since silver has enhanced absorption at this portion of the spectrum, we used $\mathrm{Al}$ for metal coating of Blu-Ray.

\section{Surface plasmons on tuned optical dises}

The effects of etching on the topography of CD surfaces are measured using an AFM (PSIA XE100), as shown in Fig. 3a. It can be seen that unetched CD surfaces have nearly rectangular grating profiles, with typical depths of $150 \mathrm{~nm}$. Since the depth is important for the 

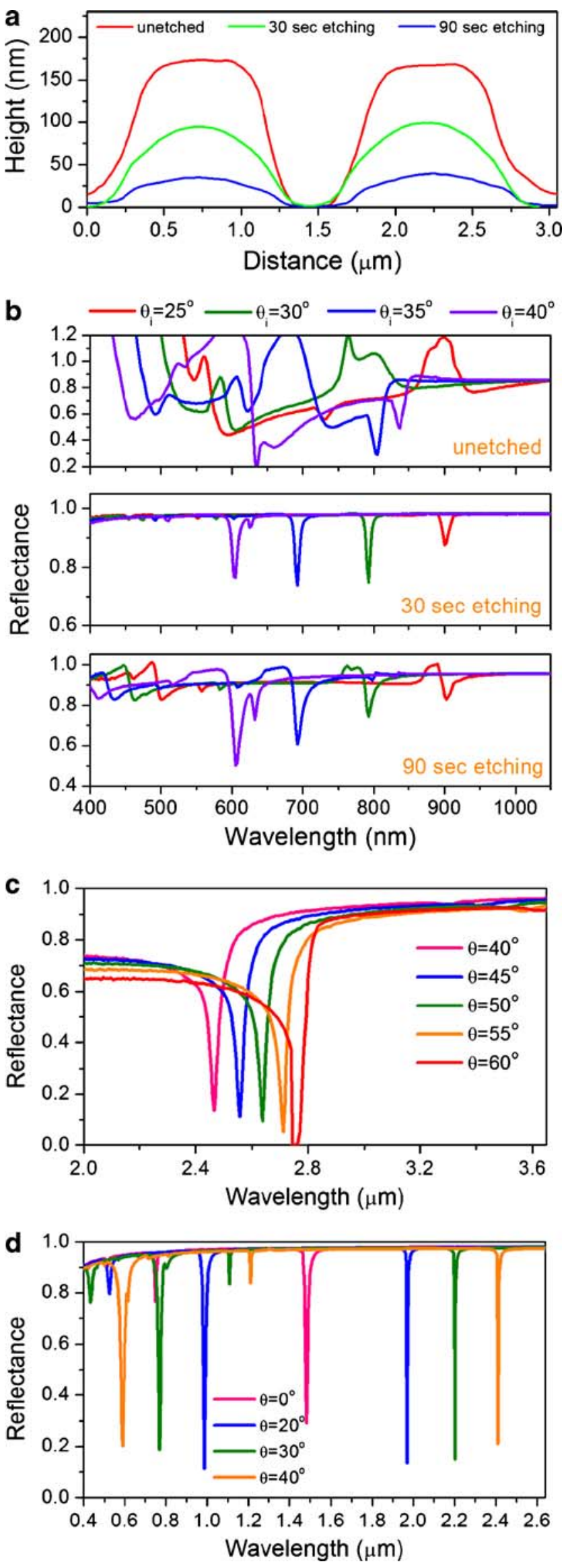

Fig. 3 a Topographic profiles of the gratings on CD-R obtained by AFM for various etching times. b Reflection spectra as a function of wavelengths for various etching times measured on the $\mathrm{CD}-\mathrm{R}$ disc coated with $50 \mathrm{~nm}$ silver. c Grating coupled plasmon resonance at infrared region observed on $\mathrm{CD}$ surfaces coated with $50 \mathrm{~nm}$ of silver, at angles of incidence of 40, 45, 50, 55 , and $60^{\circ}$. d Rigorously coupled wave analysis results for silver coated CD-R for angles of incidence of $0,20,30$, and $40^{\circ}$

operation of the $\mathrm{CD}$ as an optical disc, different brand discs have similar corrugation depths. It is observed that etching in acetone solution reduces the depth of the $\mathrm{CD}$, as well as clearing the higher frequency components of the profile and changing it to a more sinusoidal shape. Depending on the choice ambient refractive index, the angle of incidence and wavelength optimal depths for sharp and strong resonances will vary, and they must be calculated. However, typical optimal depths for visible and near infrared wavelengths lie in the vicinity of 50 to $90 \mathrm{~nm}$ for the CDs. Therefore, 30 to $90 \mathrm{~s}$ of etching was found to be adequate.

The reflection spectra are measured using spectroscopic ellipsometry (J. A. Woollam, VASE) in the visible and infrared wavelengths as shown in Fig. 3b, where progressive appearance of sharp and strong plasmon resonance peaks can be observed in the reflection spectra. For an unetched substrate, variations in depth and width along the track lines and non-sinusoidal grating profiles cause broad and not-so-well-defined resonances. However, as the surface is tuned by etching, sharper resonances appear in the reflection spectra. Also, measurements at different angles verify that the dips in the reflection spectra are due to grating coupled plasmon resonance. The plasmon resonances can also be observed in the infrared portion of the spectrum. An example of such resonances was observed using CDs, etched for $30 \mathrm{~s}$ and coated with a thin $(50 \mathrm{~nm})$ layer of silver. In this configuration, at angles of incidence of 40 to 60 degrees, plasmon resonances can be observed between 2.4 to $2.8 \mu \mathrm{m}$ with quality factors of 54 to 30 (Fig. 3c). Figure 3d shows the calculated reflection spectra for various incidence angles by using rigorously coupled wave analysis. The results agree well with measurements at visible and infrared wavelengths.

For the DVDs, the etched profiles and corresponding reflection spectra are shown in Fig. 4a. The profiles of CDs and DVDs are different in shape. For most tested CDs, the unetched profiles show nearly symmetric rectangular profile, while the DVDs have asymmetric profiles. Symmetric profile gratings as in CDs have odd spatial harmonics, whereas asymmetric profiles observed as in DVDs have both odd and even spatial harmonics. The relative intensities of harmonics change 
a

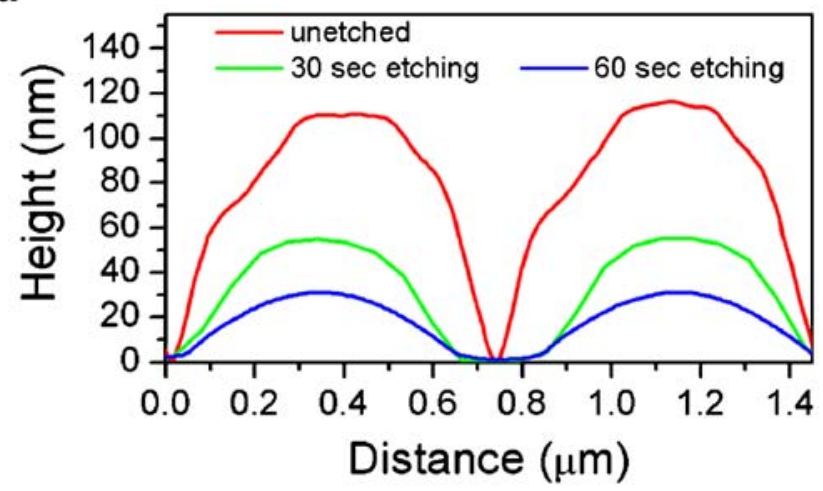

b

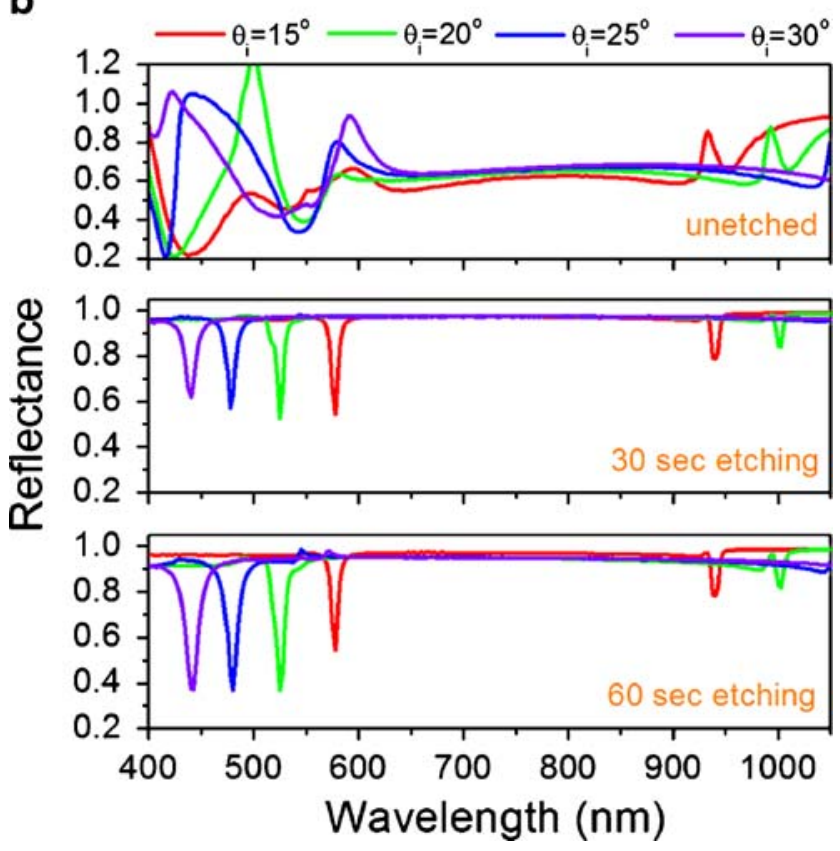

Fig. 4 a Profiles of the gratings on a DVD-R disc obtained by AFM for various etching times. b The measured reflection spectra for various incidence angles on DVD-R disc coated with $50 \mathrm{~nm} \mathrm{Ag}$

after etching; however, they are partially preserved. Such harmonics are known to modify the plasmon band structure and can also lead to plasmonic band gaps [6]. It is possible to further control the grating shape in order to control relative intensities of spatial harmonics by using techniques such as evaporation of metal at an elevated angle.

The changes in the reflection spectra as a function of wavelength for various etch depths are shown in Fig. 4b. Similar to the CD, unetched DVDs show unclear resonances at all orders, while etching significantly improves apparent quality factors of resonances. For example, the full width half maximum for 60 -s etched DVD substrates are from 5 to $25 \mathrm{~nm}$ for angles of incidence from 15 to 30 degrees. Again, experimentally observed angular dependence of the resonances agrees well with the predictions of Eq. 1.

Blu-Ray discs have significantly smaller periods than CD and DVDs, and this makes them suitable for observing plasmon resonances at smaller wavelengths. The topographic profile and reflection spectrum as measured by spectroscopic ellipsometry is given in Fig. 5. Since the excitation wavelengths were near the UV for the Blu-Ray discs, aluminum was chosen as the metal layer. The so-called wobble patterns (Fig. 5b) along the grating lines limit the quality factors [19] achievable with the untreated Blu-Ray discs; however, a small grating period allows excitation of well defined resonances in the UV. Using Al-coated Blu-Ray discs, plasmon resonances can be observed around $300 \mathrm{~nm}$ where DNA has resonant absorption (Fig. 5c). Even though measurements are incomplete due to limitation in the range of ellipsometer below $260 \mathrm{~nm}$ wavelengths, calculated results show distinct plasmonic absorption at the UV region (Fig. 5d).

\section{Observation of plasmon resonances in fluids}

Once the discs are tuned for sharp resonances, many plasmonic effects and devices based on grating coupled plasmon resonance can be demonstrated [20]. One of the most common applications of plasmon resonance is in biomolecular sensing. Such biosensors work by measuring the effective refractive index change due to molecular adsorption in an aqueous medium. Once functionalized for specific binding, the surfaces where the plasmon propagates can act as a biomolecular sensor [9]. We demonstrate such a refractive index measurement setup in a simple fluid cell as shown in Fig. 6a. The shift of the plasmon resonance can be measured in the angular or wavelength interrogation modes. Using rigorously coupled wave analysis, reflection spectra of tuned DVD gratings in a fluid cell geometry can be simulated, as is shown in Fig. 6b for water and isopropanol as fluid media for an angle of incidence $15^{\circ}$. The data shown in Fig. $6 \mathrm{~b}$ show shift of the plasmon resonance characterized by a spectroscopic ellipsometer for three different media, namely, deionized water and isopropanol. It is seen that the data are in agreement with theoretical predictions. In the device configuration shown in Fig. 6a, the refractive index measurement can be performed in the angular interrogation scheme using a simple silicon photodiode and a simple laser diode. Although less sensitive compared to prisms in a wavelength interrogation-type measurement [21], the advantage of the use of grating coupled plasmon resonance for biomolecular sensors is that high-index 
a

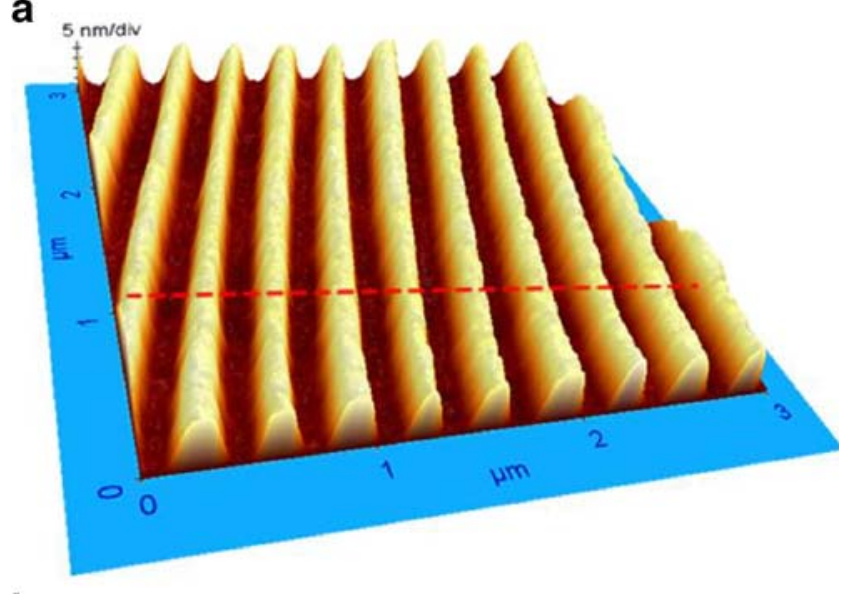

b
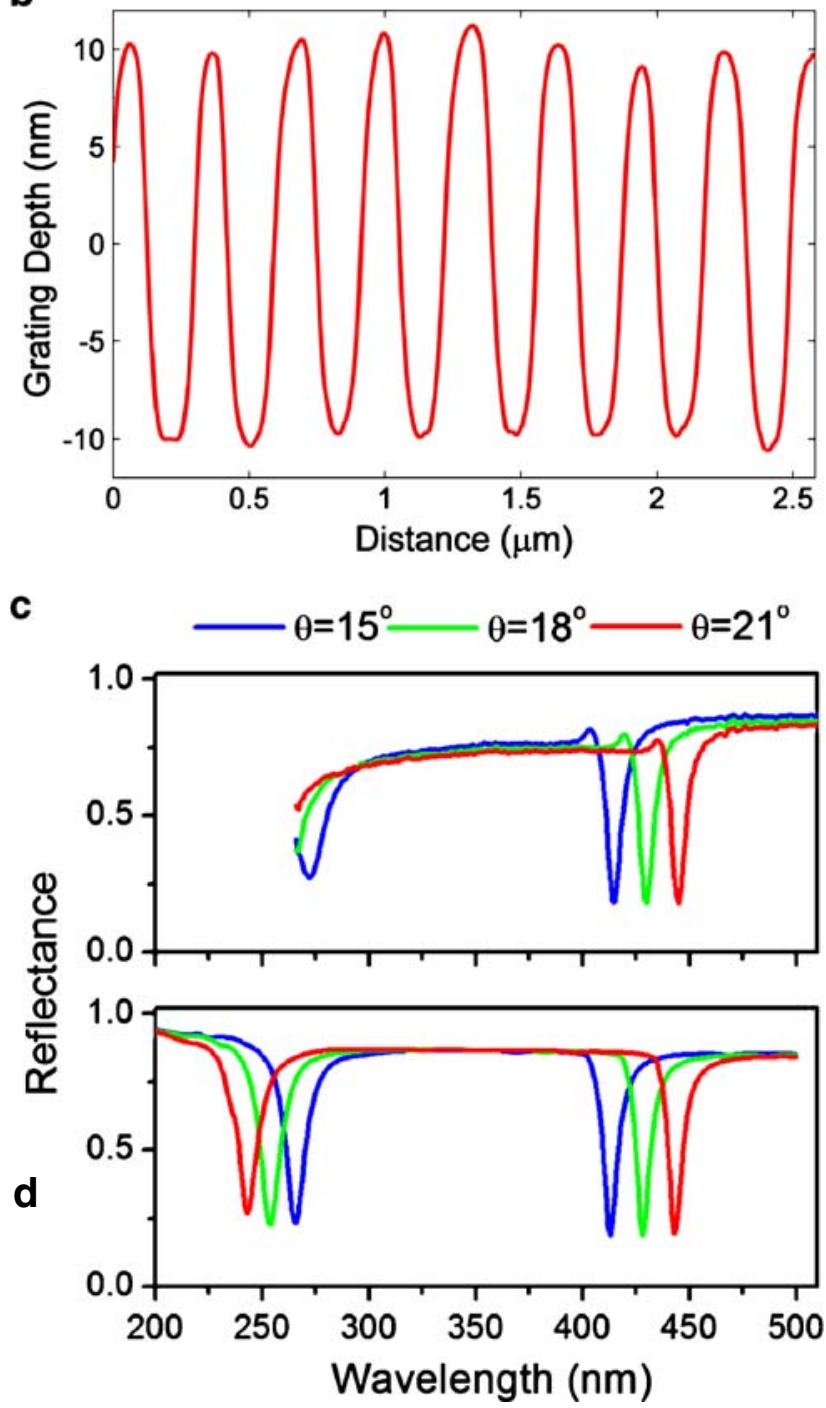

Fig. 5 a AFM micrograph showing topography of Blu-Ray disc and $\mathbf{b}$ typical line profile. $\mathbf{c}$ Reflection spectra measured on a BluRay disc coated with $50 \mathrm{~nm} \mathrm{Al}$ at angles of incidence of 15, 18, and 21 degrees. d Calculated reflection spectra agree well with measured results a
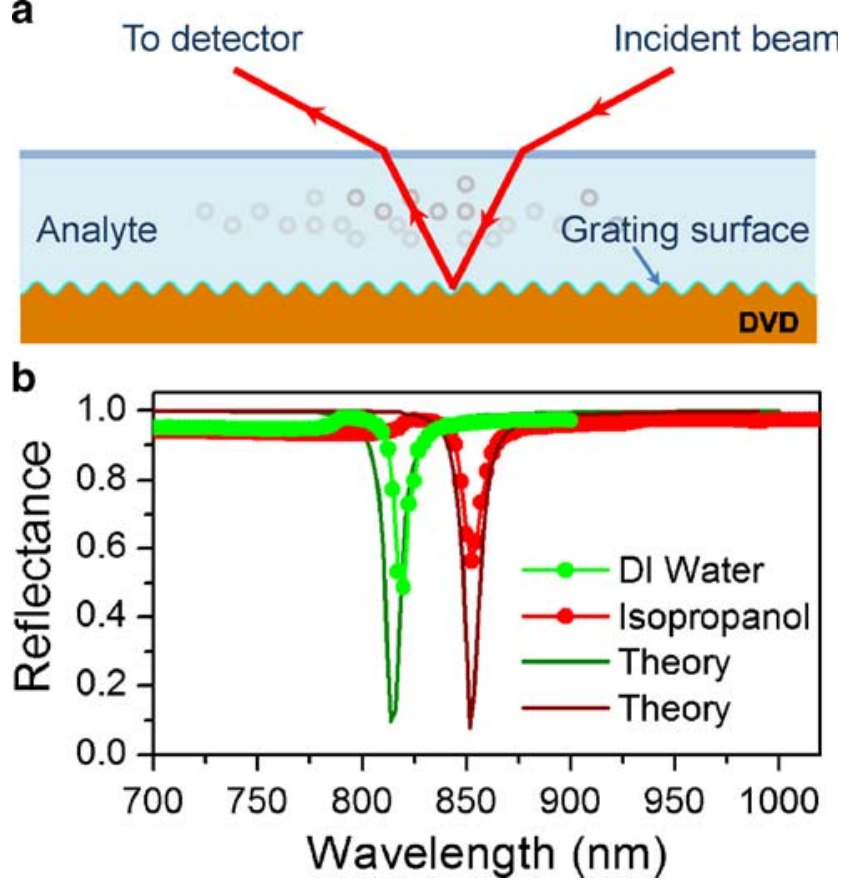

Fig. 6 a Configuration used to observe grating coupled plasmon resonance in fluid. b Theoretical prediction and experimental data for the fluid being water and isopropanol

substrates or prisms need not be used, and measurements can be performed at normal incidence. In this scheme, optical discs provide a disposable alternative for high-sensitivity plasmon resonance biomolecular sensors.

\section{Discussions}

Alternative procedures can be used to modify the topography of optical disc gratings. Heat treatment (e.g., $150^{\circ} \mathrm{C}, 5 \mathrm{~min}$ on a hotplate or $140^{\circ} \mathrm{C}, 30 \mathrm{~min}$ in an oven) was observed to reflow the grating and produce extremely smooth sinusoidal gratings of about $30 \mathrm{~nm}$ depth, adequate for sharp GCPR lines. However, unless the discs are placed on flat substrates and unless they are heated and cooled slowly, the flatness of the discs is compromised. As another alternative, oxygen plasma etching in a parallel plate PECVD system was also observed to produce optimal corrugations without disturbing the disc flatness.

A pure sinusoidal metal-coated grating has no spurious plasmon resonances and the presented procedure does not produce perfectly sinusoidal surfaces. However, the tuned gratings are shown to behave like ideal sinusoidal gratings, for a wide range of incidence angles. The deviations from ideal sinusoids can be observed in 
the reflection spectra. For example, the effect of wobble patterns and deviations from a pure sinusoid have been observed as a limitation in the peak absorption and widths of the resonances. Chemical etch tuning of the $\mathrm{CD}$ and DVDs were observed to reduce the effect of such secondary patterns. It was also observed that a low surface roughness metal coating is important for observing higher quality factors. The surface roughness of silver layers can be increased by rapid metal evaporation. However, by reducing the evaporation rate or using sputter coating, smoother silver surfaces can be obtained, increasing the quality factors of resonances.

\section{Conclusions}

In summary, we presented procedures to tune the topography of optical discs to corrugations appropriate for observing high-quality factor grating coupled plasmon resonances. As an example application, we presented refractive index measurement of a fluid, which demonstrates that such substrates can be used in disposable biosensors. The tuning of widely available discs for observation of plasmon resonance effects presents an alternative to substrates prepared by more advanced techniques such as holographic, electron beam, or focused ion beam lithography.

Acknowledgements This work is supported by TUBITAK under Project No. 106T348, 106G090, and 107T547. MB acknowledges support from the Turkish Academy of Sciences Distinguished Young Scientist Award (TUBA GEBIP). This work was performed at the UNAM-Institute of Materials Science and Nanotechnology, which is supported by the State Planning Organization of Turkey through the National Nanotechnology Research Center Project.

\section{References}

1. Homola J, Yee SS, Gauglitz G (1999) Surface plasmon resonance sensors: review. Sens Action B 54:3-15

2. Nelson BP, Grimsrud TE, Liles MR, Goodman RM, Corn RM (2001) Surface plasmon resonance imaging measurements of DNA and RNA hybridization adsorption onto DNA microarrays. Anal Chem 73(1):1-7
3. Brongersma ML, Hartman JW, Atwater HA (2000) Electromagnetic energy transfer and switching in nanoparticle chain arrays below the diffraction limit. Phys Rev B 62(24):1635616359

4. Maier SA, Atwater HA (2005) Plasmonics: localization and guiding of electromagnetic energy in metal/dielectric structures. J Appl Phys 98(1):011101

5. Naik RR, Stringer SJ, Agarwal G, Jones SE, Stone MO (2002) Biomimetic synthesis and patterning of silvernanoparticles. Nat Mater 1:169-172

6. Kocabas A, Ertas G, Senlik SS, Aydinli A (2008) Plasmonic band gap structures for surface-enhanced Raman scattering. Opt Express 16(17):12469-12477

7. Kocabas A, Senlik SS, Aydinli A (2008) Plasmonic band gap cavities on biharmonic gratings. Phys Rev B 77(19):195130

8. Zou S, Janel N, Schatz GC (2004) Silver nanoparticle array structures that produce remarkably narrow plasmon lineshapes. J Chem Phys 120(23):10871-10875

9. Homola J (2003) Present and future of surface plasmon resonance biosensors. Anal Bioanal Chem 377:528-539

10. Singh BK, Hillier AC (2006) Surface plasmon resonance imaging of biomolecular interactions on a grating-based sensor array. Anal Chem 78(6):2009-2018

11. Maier SA (2006) Plasmonics: the promise of highly integrated optical devices. IEEE J Sel Top Quant Electron 12(6):16711677

12. Barnes WL, Dereux A, Ebbesen TW (2003) Surface plasmon subwavelength optics. Nature 424:824-830

13. Raether H (1988) Surface plasmons on smooth and rough surfaces and on gratings, chapter 6. Springer-Verlag, Berlin

14. Maier SA (2007) Plasmonics: fundamentals and applications, chapter 5. Springer, New York

15. Fontana E (2004) Theoretical and experimental study of the surface plasmon resonance effect on a recordable compact disk. Appl Opt 43(1):79-87

16. Sedoglavich N, Kunnemeyer R, Talele SR, Sharpe JC (2008) Phase-polarisation contrast for surface plasmon resonance based on low cost grating substrates. Current Applied Physics 8:351-354

17. Kneipp K, Kneipp H, Itzkan I, Dasari RR, Feld MS (2002) Surface enhanced Raman scattering and biophysics. J Phys Chem 14:597-624

18. Kahl M, Voges E (2000) Analysis of plasmon resonance and surface-enhanced Raman scattering on periodic silver structures. Phys Rev B 61(20):14078-14088

19. Mank AJG, Kuiper AET, Nulens HAG, Feddes B, Wei G (2007) Detection of recording marks on digital versatile discs and blu-ray discs using conductive atomic force microscopy. Jpn J Appl Phys 46(9A):5813-5820

20. Gurel K, Kaplan B, Guner H, Bayindir M, Dana A (2009) A compact filter based on anomalous transmission in grating coupled plasmon resonance. Appl Phys Lett (in press)

21. Homola J, Koudelab I, Yee SS (1999) Surface plasmon resonance sensors based on diffraction gratings and prism couplers: sensitivity comparison. Sens Act B 54:16-24 